\title{
Sequence effects of the involuntary and the voluntary components of symbolic cueing
}

\author{
Qian Qian $^{1} \cdot$ Feng Wang ${ }^{1} \cdot$ Miao Song $^{2} \cdot$ Yong Feng $^{1} \cdot$ Keizo Shinomori $^{3}$
}

Published online: 26 December 2017

(C) The Psychonomic Society, Inc. 2017

\begin{abstract}
The orienting of attention has been found to be influenced by the previous cueing status in a spatial-cueing paradigm. The explanation for this sequence effect remains uncertain. This study separated the involuntary and the voluntary components of arrow cueing by manipulating the predicted target locations. For example, a left arrow cue may have indicated that the target was more likely to appear at the up location. Therefore, three trial types were repeated or switched between trials: cued (targets appeared along the direction of the arrows), predicted (targets appeared at the locations predicted by the arrows), and unrelated (targets appeared at the other two locations, neither cued nor predicted). RTs of cued trials were found to be significantly facilitated after a previous cued trial; however, the same effect was not observed for predicted trials. The results suggest that significant sequence effects are induced only in the involuntary component of arrow cueing. The findings support the featureintegration hypothesis for the sequence effect of symbolic cueing.
\end{abstract}

Keywords Sequence effect $\cdot$ Attention $\cdot$ Attention orienting $\cdot$ Cueing effect $\cdot$ Symbolic cueing

The perception of a sudden onset of a peripheral cue or a centrally presented symbolic cue is enough to orient our attention to the location indicated by the cue. This cuefollowing behavior has been investigated by researchers for several decades (Chica et al., 2014; Frischen et al., 2007; Posner, 2016). In the laboratory, the attention mechanisms under the cue-following behavior are usually measured using the spatial-cueing paradigm. In a typical study of this paradigm, participants are required to respond to the appearance of a target, which is preceded by a cue indicating the possible location of the target. Although traditional results in spatialcueing tasks (i.e., faster RTs in cued trials than in uncued trials) are limited within one trial, many studies have found that the performance of participants is influenced by the trial

Qian Qian

qianqian_yn@126.com

1 Yunnan Key Laboratory of Computer Technology Applications, Kunming University of Science and Technology, Kunming, China

2 Information Engineering College, Shanghai Maritime University, Shanghai, China

3 School of Information, Kochi University of Technology, Kami, Kochi, Japan types of previous trials (Dodd \& Pratt, 2007; Jongen \& Smulders, 2007; Mordkoff et al., 2008; Qian et al., 2012). Specifically, participants responded faster to targets on cued trials preceded by a cued trial relative to an uncued trial and also responded faster to targets on uncued trials preceded by an uncued trial relative to a cued trial. As a result, stronger cueing effects (i.e., the RT difference between uncued and cued trials) were induced after a preceding cued trial than after a preceding uncued trial.

However, although the sequential phenomenon in the cueing task has been proved by many studies, the rationale remains uncertain. One explanation (the so-called "automatic memory retrieval") is from the studies that used uninformative peripheral cues (Dodd \& Pratt, 2007). The main idea of this explanation is that repetition of trial types will facilitate participants' performance compared with when the trial types are switched between trials. In other words, the sequential phenomenon is described as a simple trial type repetition advantage effect.

The trial type repetition advantage hypothesis is too simple to explain the details of the sequence effect. Therefore, some later studies using uninformative arrow cues (Qian et al., 2012; Qian et al., 2012; Qian et al., 2015) prefer to explain the sequence effect with the feature-integration hypothesis (Hommel \& Colzato, 2004; Hommel et al., 2004). According to the feature-integration hypothesis, the co- 
occurrence of a cue and a target leads to a transient representation of the relation between the cue features and the target locations, thus leading to a good performance when the relation is reencountered in the next trial. The feature-integration hypothesis is more specific than the trial-type repetition advantage hypothesis, because it identifies a possible factor that may decide whether the sequence effect could occur: the easiness of the integration (Qian et al., 2017). For example, in the study by Qian et al. (2017), visually asymmetrical letter cues (e.g., the letters $d$ and $b$ ), which allow spatial correspondences between spatial features of the cue display and the target locations (Shin et al., 2011), induce stronger sequence effects than visually symmetrical letter cues (e.g., the letters $\mathrm{X}$ and $\mathrm{T}$ ). Previous studies have found significant sequence effects even with a short cue-target SOA when peripheral cues, rather than central symbolic cues (such as arrows), are tested (Mordkoff et al., 2008; Qian et al., 2012). The findings of Qian et al. (2017) provide one possible explanation for the difference in effect between the two cue types. Specifically, for peripheral cues, sequence effects can be driven by the simple integration of spatial correspondences between cue locations and target locations, which are straightforward and can be processed rapidly. However, the association between visually asymmetrical cues and target locations is slowed down, leading to an insignificant sequence effect in a relatively short SOA condition. As for the symmetrical central cues, the sequence effect is hindered by the difficulty of integration between the cues and the targets despite the length of SOAs. Another evidence that supports the feature-integration hypothesis comes from the study by Qian et al. (2015). It was found that the spatialorganization conditions of targets and cues significantly influenced the magnitude of the sequence effect.

The third explanation (the so-called "strategical adjustments") about the sequence effect in the cueing task comes from the studies that used informative arrow cues (Gomez \& Flores, 2011; Gomez et al., 2009; Jongen \& Smulders, 2007). In these studies, the arrow cues predicted target locations in most of the trials (e.g., $80 \%$ of trials were cued trials), and participants were encouraged to utilize the cues to facilitate their performance. According to the strategical-adjustments hypothesis, participants continuously adapt their utilization of the cue depending on whether the cues correctly or incorrectly direct their attention on the previous trial, leading to a stronger cueing effect after a cued trial than after an uncued trial.

Although the results of the cueing studies with uninformative cues (Qian et al., 2012) may have proved that sequence effects can be induced implicitly and involuntarily, we still do not known whether the voluntary control of the participants in cueing tasks can induce sequence effects. The difficulty in answering this question results from the fact that the involuntary cueing component was usually confounded with the voluntary cueing component in prior studies that used informative cues. For example, a left-pointing arrow predicted a left target in most trials in the study by Jongen and Smulders (2007). However, simply presenting an uninformative arrow cue to the participants was enough to orient their attention to the pointing location of the arrow (Hommel et al., 2001). Therefore, the cueing and the sequential results observed in the study by Jongen and Smulders (2007) may have originated from both the involuntary and the voluntary components of the cueing.

In the present central-symbolic-cueing study, the contribution of the involuntary and the voluntary components of the arrow cueing to the sequence effect was systematically investigated. In Experiment 1, the possible target locations were increased (i.e., up, down, left, and right) while pointing directions of arrow cues remained the same (i.e., left and right). Importantly, the arrow cues predicted the possible target locations that were along a different axis from the arrow directions (i.e., a left arrow may have indicated that the target had a higher probability of appearing at the up location than at the other locations). As a result, three kinds of cue types were included: cued (targets appeared on the same location as the cue direction, such as a left cue with a left target), predicted (targets had a high possibility of appearing at one of the locations adjacent to the cue direction, such as a left cue with an up target), and unrelated (targets appeared at the one of the two other locations, such as a left cue with a right target or a left cue with a down target). Thus, it was possible to measure the involuntary (between cued and unrelated trials) and the voluntary (between predicted and unrelated trials) components of the arrow cueing separately within the same experiment and with the same participants. The trial-type repetition advantage hypothesis would have predicted significant sequence effects in both the involuntary and the voluntary components of the cueing, because trial-type repetitions existed in both conditions. On the other hand, the feature-integration hypothesis would have predicted a significant sequence effect only in the involuntary cueing component. As noted, this is because feature integration is most likely to occur when spatial correspondences exists between the cues and the targets (Qian et al., 2017). For example, the integration may occur between a left target and a left-pointing arrow, but such integration is difficult between an up target and a left-pointing arrow. Finally, the strategical-adjustments hypothesis would have predicted a significant sequence effect only in the voluntary cueing component. In Experiment 2, the possible target locations were up and down and the pointing directions of the arrow cues were left and right. Therefore, only the voluntary component of the cueing was measured.

\section{Experiment 1}

\section{Participants}

A total of 30 students (with a mean age of 25 years, range 19-31 years, 13 females) consented to participate in this experiment. All 
participants reported normal or corrected-to-normal vision and were naive to the purpose of the experiment.

\section{Apparatus}

The stimuli were presented on an LCD display operating at a $60-\mathrm{Hz}$ frame rate. The participants were seated approximately $57-\mathrm{cm}$ away from the screen in a dimly lit room. A chinrest was used to prevent any unnecessary head movements.

\section{Stimuli}

A cross, subtending $1.5^{\circ}$, was placed at the center of the screen as a fixation point. The central cue was an arrow. The central horizontal line of the arrow was $3.5^{\circ}$ in length. An arrow head and an arrow tail were displayed at the ends of the central line, pointing to the left or right locations. The length of an arrow from the tip of the arrow head to the ends of the tail was $4.5^{\circ}$. The target stimulus was a capital letter $\mathrm{X}$ measuring $1^{\circ}$ wide and $1^{\circ}$ high, and it was presented $12^{\circ}$ away from the fixation point to the top, bottom, left, or right of the screen. There were no placeholders for the four possible target locations. The stimuli were all black and were presented on a light-gray background.

\section{Design}

The cue-target SOA was $600 \mathrm{~ms}$. On each trial, the cue directions were selected pseudo-randomly. When the cue direction pointed to the left, the probability that the target would appear at the up location (or at the down location, for half of the participants) was four times the probability that the target would appear at one of the other three locations; when the cue direction pointed to the right, the probability that the target would appear at the down location (or at the up location, for half of the participants) is four times the probability that it would appear at one of the other three locations. Therefore, in each trial, the probability that the target would appear at the predicted location (up or down) and one of the three other locations was approximately $57 \%$ and $14 \%$, respectively. There were 12 blocks (divided into 2 sessions) with 96 trials each. The participants took a long break between sessions and a short break between blocks. Twelve trials in each block were catch trials in which the target did not appear. The participants were instructed not to respond if the target did not appear. Including 20 training trials, there were in all 1,172 trials for each participant. The RTs of the first trials on each block and the RTs of the trials followed a catch trial were excluded from the analysis.

The sequence effects were first tested in a two-way analysis of variance (ANOVA) with the within-participant factors of the previous cue type (pre-cued, pre-predicted, or pre-unrelated) and the current cue type (cued, predicted, or unrelated) on RTs. A significant interaction between the previous cue type and the current cue type would represent a significant sequence effect between trials. After that, paired-samples $t$ tests were used to analyze the involuntary cueing component by comparing the RTs among pre-cued $\rightarrow$ cued, pre-unrelated $\rightarrow$ cued, and pre-predicted $\rightarrow$ cued trial sequences. Facilitated RTs in pre-cued $\rightarrow$ cued trial sequences would indicate a significant sequence effect in the involuntary cueing component. A similar analysis would be conducted for the voluntary cueing component by comparing the RTs among pre-predicted $\rightarrow$ predicted, pre-cued $\rightarrow$ predicted, and pre-unrelated $\rightarrow$ predicted trial sequences. Facilitated RTs in pre-predicted $\rightarrow$ predicted trial sequences would indicate a significant sequence effect in the voluntary cueing component.

\section{Procedure}

An illustration of the sequence of events can be seen in Figure 1. Participants were instructed to keep fixating on the center of the screen. First, a fixation cross appeared at the center of the screen for $1,000 \mathrm{~ms}$, after which the cue stimulus appeared. After the 600 -ms cue-target SOA, a target letter X appeared at one of the four possible locations until participants had responded or 1,200 ms had elapsed. The cue stimulus remained on the screen after the appearance of the target. Participants were instructed to respond when the target appeared by pressing the SPACE key with the index finger of their right hand as quickly and accurately as possible. Participants also were informed that the central stimuli predicted the location at which the target would appear and that they should try to utilize the central cues to facilitate their performance.

\section{Results}

Errors The participants missed an average of approximately $0.3 \%$ of the targets and made false alarm errors on approximately $3.4 \%$ of the catch trials. Anticipations (RTs of less than 100 $\mathrm{ms}$ ) and outliers (RTs over 1,000 ms) were classified as errors and were excluded from further analysis. As a result, approximately $0.8 \%$ of all trials were excluded as errors. An ANOVA with the within-participant factors of the previous cue type and the current cue type was conducted on the percent errors. None of the factors or interactions was significant ( $p s>0.25$ ).

RTs A pre-analysis confirmed that the RTs under the two target location conditions for unrelated trials (i.e., targets appeared on the opposite side of the cued or the predicted locations) were not significantly different from each other $(p=0.621)$, so the RTs of these two conditions were not divided and the analysis mentioned above was followed. The average RTs under different conditions are shown in Table 1 and Figure 2. An ANOVA with the withinparticipant factors of the previous cue type and the current cue type was conducted on RTs. There was a significant effect of the previous cue type, $\mathrm{F}(2,58)=3.967, p=0.024$, indicating that the RTs were shorter in the pre-cued condition than in the other two conditions. The main effect of the current cue type also was significant, F $(1.047,30.361)=4.683, p=0.037$ (Greenhouse- 


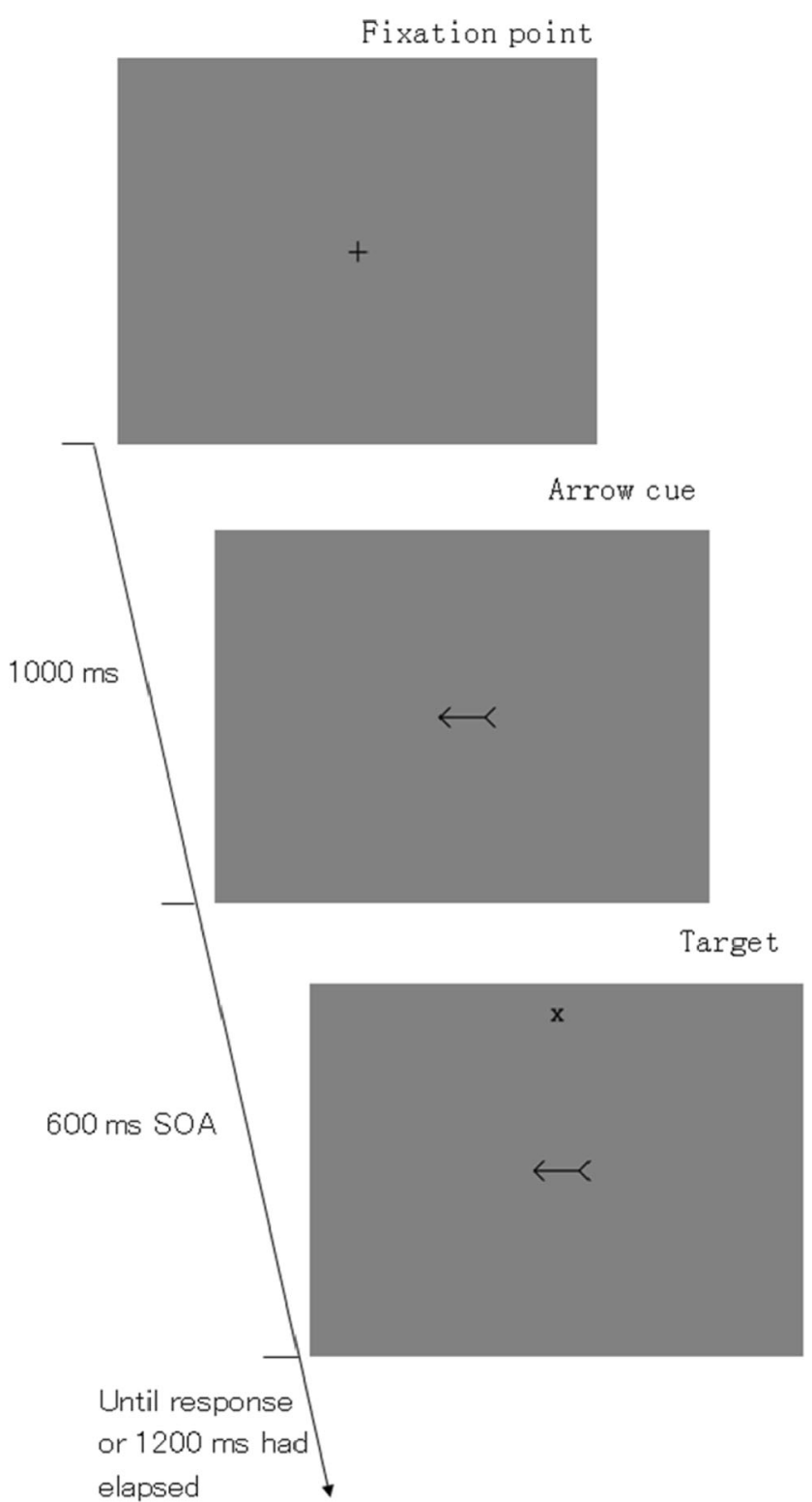

Fig. 1 Illustration of the sequence of events in Experiment 1. When the left arrow predicts an up target, the trial in the figure (left arrow with up target) is a predicted trial. The trial (left arrow with left target) will be a cued trial. The trial (left arrow with down or right target) will be an unrelated trial

Geisser adjusted, due to significant Mauchly's Test of Sphericity), reflecting that the RTs were shorter in the predicted condition than in the other two conditions. In addition, the interaction between the previous cue type and the current cue type was significant, $\mathrm{F}(4,116)=8.599, p<0.001$, indicating that there were significant sequence effects between trials. To further investigate the details of the sequence effect, paired-samples $t$ tests were conducted on the RTs of each trial sequence. It was found that the RTs of pre-cued $\rightarrow$ cued trial sequences were significantly faster than those of pre-unrelated $\rightarrow$ cued and pre-predicted $\rightarrow$ cued trial sequences [ $\mathrm{t}(29)=3.900, p=0.001$, and $\mathrm{t}(29)=$ $4.883, p<0.001$, respectively], indicating that significant
Table 1. The mean RTs (accurate to bits) and errors (accurate to the first decimal place) under different conditions in Experiment 1

\begin{tabular}{llll}
\hline & Pre-cued & Pre-unrelated & Pre-predicted \\
\hline Cued & $388(18)$ & $403(18)$ & $406(19)$ \\
& $0.9 \%$ & $0.8 \%$ & $0.8 \%$ \\
Unrelated & $411(19)$ & $407(18)$ & $412(19)$ \\
& $1.3 \%$ & $0.9 \%$ & $0.9 \%$ \\
Predicted & $373(8)$ & $373(9)$ & $369(8)$ \\
& $0.6 \%$ & $0.5 \%$ & $0.7 \%$ \\
\hline
\end{tabular}

Standard deviations for each condition are shown in brackets

sequence effects were induced in the involuntary cueing component. On the other hand, the RTs of pre-predicted $\rightarrow$ predicted trial sequences were not significantly different from those of pre-unrelated $\rightarrow$ predicted or pre-cued $\rightarrow$ predicted trial sequences $[\mathrm{t}(29)$ $=1.471, p=0.152$, and $\mathrm{t}(29)=1.174, p=0.25]$. In addition, similar comparisons were run among pre-cued $\rightarrow$ unrelated, preunrelated $\rightarrow$ unrelated, and pre-predicted $\rightarrow$ unrelated trial sequences, but no significant differences were found $(p s>0.05)$. Therefore, the results showed that significant sequence effects were only found in the involuntary cueing component and were expressed as facilitated RTs in pre-cued $\rightarrow$ cued trial sequences.

\section{Experiment 2}

The results of Experiment 1 did not show significant sequence effects in the voluntary cueing component. However, before we conclude that voluntary control does not induce sequence effects, a shortcoming in the previous experimental design should be mentioned. The probability that the target would appear at the predicted location was only $57 \%$ in Experiment 1, but the probability usually reaches $80 \%$ in traditional cueing tasks with informative cues. The relatively small number of predicted trials among all trials may have

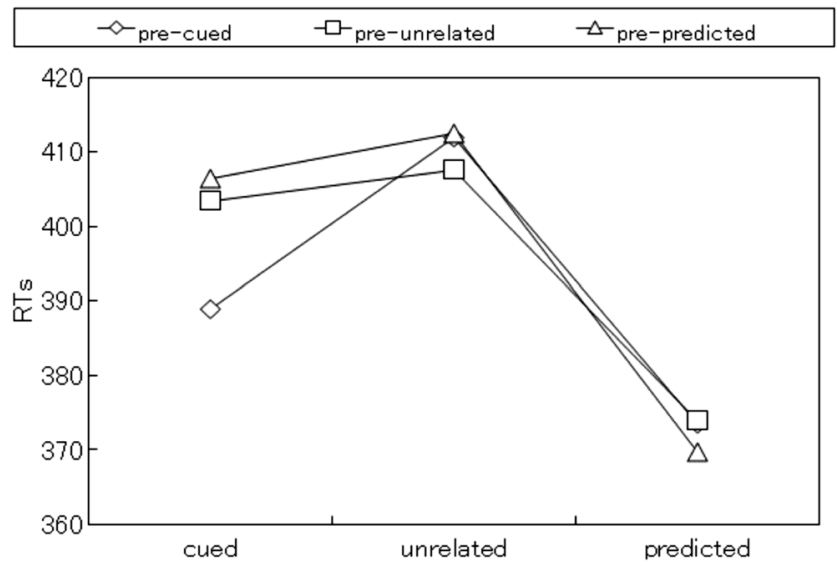

Fig. 2 The average RTs under different previous and current trial types in Experiment 1 
hindered the participants' intention to utilize the cues, resulting in nonsignificant sequence effects in the voluntary cueing component. To avoid this shortcoming, the ratio of predicted trials among all trials was increased by reducing the possible target locations and only the voluntary cueing component was measured in the Experiment 2.

\section{Participants}

A total of 16 students (with a mean age of 23 years, range 2226 years, 11 females) consented to participate in this experiment. All participants reported normal or corrected-to-normal vision and were naive to the purpose of the experiment.

\section{Apparatus, stimuli, design, and procedure}

The apparatus, stimuli, design, and procedure were the same as those in Experiment 1 except for the possible target locations (i.e., only up and down). When the cue direction pointed to the left, the probability that the target would appear at the up location (or the down location, for half of the participants) was four times the probability of the target appearing at the down location (or the up location, for half of the participants). When the cue direction pointed to the right, the probability that the target would appear at the down location (or the up location, for half of the participants) was four times than its probability of appearing at the up location (or the down location, for half of the participants). Therefore, on each trial, the probabilities that the target would appear at the predicted location and the unpredicted location were $80 \%$ and $20 \%$, respectively. There were 6 blocks with 120 trials each. In each block, 20 trials were catch trials. Including 20 training trials, there were a total of 740 trials for each participant.

The sequence effects were tested in a two-way analysis of variance (ANOVA) with the within-participant factors of the previous cue type (pre-predicted, pre-unpredicted) and the current cue type (predicted, unpredicted) on the RTs. A significant interaction between the previous cue type and the current cue type would represent a significant sequence effect between trials.

\section{Results}

Errors The participants missed an average of about $0.09 \%$ of the targets and made false-alarm errors on approximately $3 \%$ of the catch trials. Anticipations (RTs of less than $100 \mathrm{~ms}$ ) and outliers (RTs over 1,000 ms) were classified as errors and were excluded from further analysis. As a result, approximately $0.7 \%$ of all trials were excluded as errors. An ANOVA with the within-participant factors of the previous cue type and the current cue type was conducted on the percent errors. None of the factors or interactions was significant ( $p \mathrm{~s}>0.54)$.
RTs The average RTs under different conditions are shown in Table 2 and Figure 3. An ANOVA with the within-participant factors of the previous cue type and the current cue type was conducted on the RTs. There was a main effect of the current cue type, $\mathrm{F}(1,15)=6.105, p=0.026$, indicating that the RTs were shorter in a predicted condition than in an unpredicted condition. Importantly, the interaction between the previous cue type and the current cue type was not significant, $\mathrm{F}(1,15)$ $=1.411, p=0.253$, reflecting that no significant sequence effects were found for the voluntary cueing component, which was similar to the findings of Experiment 1 . To further confirm that the findings of the two experiments were similar, an ANOVA with the between-participant factor of the experiment (Exp1, Exp2) and the within-participant factors of the previous cue type (pre-predicted, pre-unpredicted) and the current cue type (predicted, unpredicted) was conducted on the corresponding RTs (notice that the data of Exp1 only included the trials with the exact same layout as the trials of Exp2). There was a main effect of the current trial type, $\mathrm{F}(1,44)=11.413, p$ $=0.002$, indicating that the RTs were shorter in a predicted condition than in an unpredicted condition. None of the other factors or interactions was significant $(p s>0.06)$.

\section{Discussion}

The findings described in the present study have important implications for the widely reported trial-by-trial modulations of attention mechanisms in the cueing paradigm. Indeed, the sequence effect in the cueing tasks has been explained as repetition advantage of trial types, feature integration between cue features and target locations, or strategical adjustments of cue usability. The strategical-adjustments hypothesis is mainly proposed by the studies with informative arrow cues (Gomez \& Flores, 2011; Gomez et al., 2009). Because the predicted target locations were usually the same as the pointing location of the arrows, the results of these studies may have confounded the involuntary and the voluntary components of the arrow cueing. By increasing the possible target locations and manipulating the predicted target locations, the present study separated the involuntary and the voluntary cueing components. The results of Experiment 1 show that the significant sequence effect is only found in the

Table 2. The mean RTs (accurate to bits) and errors (accurate to the first decimal place) under different conditions in Experiment 2

\begin{tabular}{lll}
\hline & Pre-predicted & Pre-unpredicted \\
\hline Predicted & $364(15)$ & $370(15)$ \\
& $0.5 \%$ & $0.6 \%$ \\
Unpredicted & $422(27)$ & $419(27)$ \\
& $0.6 \%$ & $0.5 \%$ \\
\hline
\end{tabular}

Standard deviations for each condition are shown in brackets 


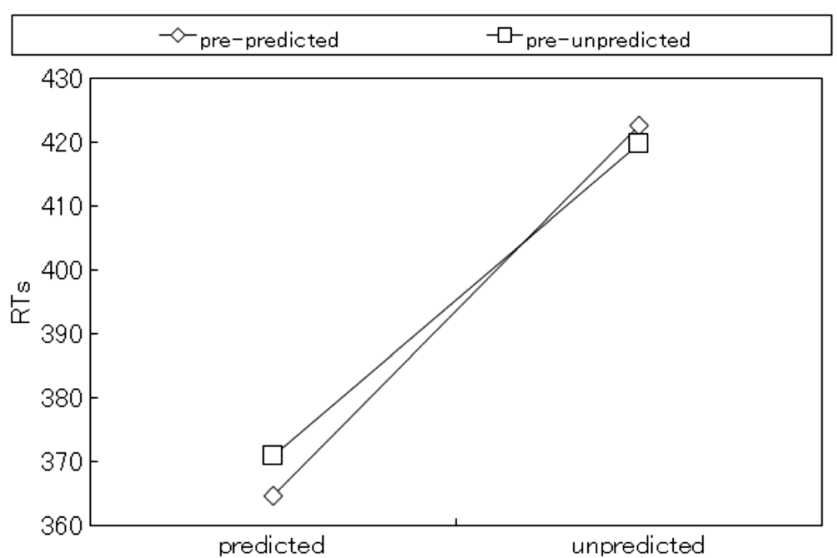

Fig. 3 The average RTs under different previous and current trial types in Experiment 2

involuntary cueing component (i.e., in pre-cued $\rightarrow$ cued trial sequences), but not in the voluntary cueing component (i.e., in prepredicted $\rightarrow$ predicted trial sequences). By ruling out the influence of the relatively low ratio of the predicted trials among all trials in Experiment 1, the results of Experiment 2 confirmed that the voluntary control of the participants in cueing tasks did not induce sequence effects.

By using uninformative central cues, several previous studies have proved that sequence effects in arrow cueing tasks can be induced implicitly and involuntarily (Qian et al., 2012; Qian et al., 2012; Qian et al., 2015). However, none of the previous studies can answer the question raised in the present study: can the voluntary control of the participants induce sequence effects? Based on the findings of the present study, the answer is probably "No." Furthermore, the present study had two advantages in methodology. First, in Experiment 1, the involuntary and the voluntary components of the arrow cueing were separated but still co-existed within one experiment. Therefore, the results could not be attributed to any differences that may have existed between different experiments and between different participants. Second, the results of Experiment 2 proved that the findings could not be attributed to the reduced predictive power of the cues.

The present results do not support the strategical-adjustments hypothesis since there is no significant sequence effect in the prepredicted $\rightarrow$ predicted trial sequences. In addition, the trial type repetition advantage hypothesis is unable to explain the whole results. According to this hypothesis, the sequence effect should exist whenever the trial types repeated between trials. However, the present results show that significant sequence effects only appeared in the pre-cued $\rightarrow$ cued trial sequences, not in the prepredicted $\rightarrow$ predicted trial sequences. The present results can be well explained by the feature-integration hypothesis. Specifically, in pre-cued $\rightarrow$ cued trial sequences, the cooccurrence of the cue and the target forms a spatial correspondence relationship between them (e.g., a left cue with a left target). This spatial relationship is completely repeated (e.g., a left cue with a left target again) or alternated (e.g., a right cue with a right target) when the trial types are repeated between trials, but it is only partially repeated when the trial types are alternated in an unrelated or a predicted trial. As a result, the RTs of cued trials are facilitated and the RTs of unrelated or predicted trials are prolonged after a preceding cued trial. Furthermore, since the pointing arrows and the target locations are along different axis in pre-predicted $\rightarrow$ predicted trial sequences, the spatial correspondence relationship cannot be easily formed (Qian et al., 2017), leading to insignificant sequence effects in this condition.

It is important to note that although significant cueing effects were observed in the involuntary component of the current cueing task, it does not imply that the observed cueing effects and sequence effects are under the control of the reflexive and automatic shift of attention. Indeed,several studies have suggested that uninformative arrow cues do not produce a pure automatic and reflexive orienting (Green \& Woldorff, 2012; Ristic \& Kingstone, 2012) . Another piece of evidence against the reflexive notion for the sequence effect of arrow cueing is that the sequence effect was only observed when the SOA on the preceding trial was relatively long (Qian et al., 2012). Fortunately, the exact orienting mechanisms under the arrow cueing do not affect the validity of the featureintegration hypothesis. The important thing about the hypothesis is the spatial correspondence between cues and targets. For now, we can say that the sequence effect is probably a common phenomenon in the cueing paradigm and it works in an implicit manner without voluntary intervention.

Significant influence of previous trial status on the performance of current trials also has been reported in other paradigms. For example, in the visual search paradigm, it was found that participants' RTs in current trials are facilitated when the target feature (e.g., color, location, orientation, or shape) is the same as that used in the preceding trials (Maljkovic \& Nakayama, 2000; Peremen, Hilo, \& Lamy, 2013). This sequence effect in visual search is usually called "priming" and is generally believed to be afforded by implicit visual memory mechanisms (Kristjánsson, 2006). Both the priming effect and the sequence effect in cueing tasks can happen implicitly and do not need voluntary intervention. Similar trial-by-trial influences were found in other attentional tasks, such as the Flanker, the Stroop, and the Simon tasks (Duthoo, Abrahamse, Braem, Boehler, \& Notebaert, 2014; Egner, 2007; Hommel et al., 2004). One major difference between these tasks and the cueing tasks is that they reflect different attention processes: executive control and orienting, respectively. Nevertheless, similar sequence effects are found in all these tasks. Overall, sequence effects between trials seem to be a common phenomenon in human visual perception. Further investigations about the similarities and the differences of the sequence effect among these different paradigms may shed new light on human attention and memory mechanisms. 
In summary, the present study demonstrated that the sequence effects of symbolic cueing were only induced in the involuntary cueing component, rather than in the voluntary cueing component. Overall, our results support the featureintegration hypothesis for sequential processing in the symbolic cueing paradigm.

Acknowledgements This research is supported by the National Science Foundation of China (31300938, 61462053, 61662042, and 61403251), the Natural Science Foundation of Yunnan Province of China (2016FB107), and the KUT President Discretionary Support-Grant to KS.

\section{References}

Chica, A. B., Martin-Arevalo, E., Botta, F., \& Lupianez, J. (2014). The Spatial Orienting paradigm: how to design and interpret spatial attention experiments. Neuroscience and Biobehavioral Reviews, 40, 35-51. doi: https://doi.org/10.1016/j.neubiorev.2014.01.002

Dodd, M. D., \& Pratt, J. (2007). The effect of previous trial type on inhibition of return. Psychological Research, 71(4), 411-417. doi: https://doi.org/10.1007/s00426-005-0028-0

Duthoo, W., Abrahamse, E. L., Braem, S., Boehler, C. N., \& Notebaert, W. (2014). The heterogeneous world of congruency sequence effects: an update. Frontiers in Psychology, 5, 1001. doi: https://doi. org/10.3389/fpsyg.2014.01001

Egner, T. (2007). Congruency sequence effects and cognitive control. Cognitive, Affective, \& Behavioral Neuroscience, 7(4), 380-390.

Frischen, A., Bayliss, A. P., \& Tipper, S. P. (2007). Gaze cueing of attention: visual attention, social cognition, and individual differences. Psychological Bulletin, 133(4), 694-724. doi: https://doi.org/10. 1037/0033-2909.133.4.694

Gomez, C. M., \& Flores, A. (2011). A neurophysiological evaluation of a cognitive cycle in humans. Neuroscience and Biobehavioral Reviews, 35(3), 452-461. doi: https://doi.org/10.1016/j.neubiorev. 2010.05.005

Gomez, C. M., Flores, A., Digiacomo, M. R., \& Vazquez-Marrufo, M. (2009). Sequential P3 effects in a Posner's spatial cueing paradigm: trial-by-trial learning of the predictive value of the cue. Acta Neurobiologiae Experimentalis (Wars), 69(2), 155-167.

Green, J. J., \& Woldorff, M. G. (2012). Arrow-elicited cueing effects at short intervals: Rapid attentional orienting or cue-target stimulus conflict? Cognition, 122(1), 96-101. doi: https://doi.org/10.1016/j. cognition.2011.08.018
Hommel, B., \& Colzato, L. (2004). Visual attention and the temporal dynamics of feature integration. Visual Cognition, 11(4), 483-521.

Hommel, B., Pratt, J., Colzato, L., \& Godijn, R. (2001). Symbolic control of visual attention. Psychological Science, 12(5), 360-365.

Hommel, B., Proctor, R. W., \& Vu, K. P. (2004). A feature-integration account of sequential effects in the Simon task. Psychological Research, 68(1), 1-17. doi: https://doi.org/10.1007/s00426-003-0132-y

Jongen, E. M., \& Smulders, F. T. (2007). Sequence effects in a spatial cueing task: endogenous orienting is sensitive to orienting in the preceding trial. Psychological Research, 71(5), 516-523. doi: https://doi.org/10.1007/s00426-006-0065-3

Kristjánsson, Á. (2006). Rapid learning in attention shifts: A review. Visual Cognition, 13(3), 324-362.

Maljkovic, V., \& Nakayama, K. (2000). Priming of popout: III. A shortterm implicit memory system beneficial for rapid target selection. Visual Cognition, 7(5), 571-595.

Mordkoff, J. T., Halterman, R., \& Chen, P. (2008). Why does the effect of short-SOA exogenous cuing on simple RT depend on the number of display locations? Psychonomic Bulletin \& Review, 15(4), 819-824. doi: https://doi.org/10.3758/pp.15.4.819

Peremen, Z., Hilo, R., \& Lamy, D. (2013). Visual consciousness and intertrial feature priming. Journal of Vision, 13(5), 1. doi: https:// doi.org/10.1167/13.5.1

Posner, M. I. (2016). Orienting of attention: Then and now. The Quarterly Journal of Experimental Psychology (Hove), 69(10), 1864-1875. doi: https://doi.org/10.1080/17470218.2014.937446

Qian, Q., Shinomori, K., \& Song, M. (2012). Sequence effects by nonpredictive arrow cues. Psychological Research, 76(3), 253-262. doi: https://doi.org/10.1007/s00426-011-0339-2

Qian, Q., Song, M., Shinomori, K., \& Wang, F. (2012). The functional role of alternation advantage in the sequence effect of symbolic cueing with nonpredictive arrow cues. Attention, Perception, \& Psychophysics, 74(7), 1430-1436. doi: https://doi.org/10.3758/ s13414-012-0337-5

Qian, Q., Wang, F., Feng, Y., \& Song, M. (2015). Spatial organisation between targets and cues affects the sequence effect of symbolic cueing. Journal of Cognitive Psychology, 27(07), 855-865. doi: https://doi.org/10.1080/20445911.2015.1048249

Qian, Q., Wang, F., Song, M., Feng, Y., \& Shinomori, K. (2017). Spatial Correspondence Learning is Critical for the Sequence Effects of Symbolic Cueing. Japanese Psychological Research, 59(3), 209-220.

Ristic, J., \& Kingstone, A. (2012). A new form of human spatial attention: Automated symbolic orienting. Visual Cognition, 20(3), 244-264. doi: https://doi.org/10.1080/13506285.2012.658101

Shin, M.-J., Marrett, N., \& Lambert, A. J. (2011). Visual orienting in response to attentional cues: Spatial correspondence is critical, conscious awareness is not. Visual Cognition, 19(6), 730-761. doi: https://doi.org/10.1080/13506285.2011.582053 